\title{
Grey Level Image Components for Multi-scale Representation
}

\author{
Giuliana Ramella and Gabriella Sanniti di Baja \\ Istituto di Cibernetica E. Caianiello, CNR, Via Campi Flegrei 34, \\ 80078, Pozzuoli (Naples), Italy \\ (g.ramella,g.sannitidibaja)@cib.na.cnr.it
}

\begin{abstract}
A method to identify grey level image components, suitable for multi-scale analysis, is presented. Generally, a single threshold is not sufficient to separate components, perceived as individual entities. Our process is based on iterated identification and removal of pixels, with different grey level values, causing merging of grey level components at the highest resolution level. A growing process is also performed to restore pixels far from the fusion area, so as to preserve as much as possible shape and size of the components. In this way, grey level components can be kept as separated also when lower resolution representations are built, by means of a decimation process. Moreover, the information contents of the image, in terms of shape and relative size of the components, is preserved through lower resolution representations, compatibly with the resolution.
\end{abstract}

\section{Introduction}

Grey level images are of large use in image analysis tasks. One of the main problems that have to be faced is image segmentation, necessary to distinguish foreground components from the background. The method to be used depends on problem domain. The easiest way, unfortunately seldom effective, is to fix a threshold and to assign to the foreground all pixels with grey level larger than the threshold, and to the background all remaining pixels, e.g., [1]. The result of this process is, generally, an image with a number of components different from the expected one. Actually, the threshold should assume different values in different parts of the image, to allow correct identification of foreground components. These methods are generally referred to as multi-threshold methods, e.g., [2,3]. An unwanted side effect of thresholding is that the size of foreground components is likely to be significantly reduced with respect to the perceived size. In fact, the same grey level distribution can characterise both the fusion area among foreground components perceived as individual entities, and (peripheral) parts of foreground components, located far from the fusion area. More sophisticated techniques, especially based on watershed transformation, generally produce better result [4-6]. These techniques, however, need a complex preliminary phase to identify the sources for watershed segmentation, as well as an equally complex post-processing phase necessary to reduce the unavoidable over-segmentation.

The method that we propose in this paper is a small addition to the literature on image segmentation. It is based on non-topological erosion and topological expansion, is computationally non expensive and in the experiments we have done performed quite well. Our method can be classified as a multi-threshold method. In fact, once the minimum and the maximum possible values of the threshold have been 
fixed, depending on problem domain, the threshold is automatically increased and the thresholding process is repeated. An advantage with respect to other multi-threshold methods is that we avoid an excessive reduction of the size of foreground components. In fact, after each thresholding that actually produces a separation of foreground components, a growing process is accomplished, to restore pixels of the foreground components far from the fusion area. Our method has proved to be useful especially when a multi-scale approach to image analysis is followed. If a lower resolution image is obtained, by applying a decimation process to the non-segmented initial image, perceived individual grey level components tend to merge, producing one agglomerate where individual components are no longer distinguishable, especially at low resolution. In turn, if a decimation process is applied to a segmented image and care is taken to avoid merging of individual components, shape information is better preserved also at low resolution. We will show the performance of our segmentation method in the framework of multi-scale image analysis. To this purpose, we will illustrate a decimation process able to prevent merging among individual components, within the limits of decreasing resolution. To preserve topology through lower resolutions, we first identify and single out in the input image all significant foreground components. Then, after each decimation process, we use the segmented high resolution initial image as a reference set and open canals so as to separate foreground parts that constituted individual entities at the highest resolution.

\section{Detecting Grey Level Components}

Let $\mathrm{G}$ be the input grey level image, and let $\mathrm{g}$ denote any pixel of $\mathrm{G}$ as well as its grey level value. A starting value for the threshold, $\theta_{i}$, needs to be fixed, depending on grey level distribution in the image. The value of $\theta_{i}$ should be such that all pixels with grey level $g \leq \theta_{\mathrm{i}}$ can, without any doubt, be interpreted correctly as background pixels. A final value for the threshold, $\theta_{\mathrm{f}}$, needs also to be fixed. This value should be such that all pixels with grey level $\mathrm{g}>\theta_{\mathrm{f}}$ certainly belong to some component, perceived as a foreground component. In the most general case, the initial value $\theta_{i}$ can be set to the minimal grey level found in the image and the final value $\theta_{\mathrm{f}}$ can be set to the maximal grey level, decreased by 1 . Of course, the initial and final values of the threshold should be more carefully set, depending on user's needs. In particular, a very small initial value could cause the detection of a number of noisy components, erroneously interpreted as belonging to the foreground. In turn, the final value should be set in such a way to prevent excessive foreground fragmentation, which would split foreground components perceived as individual entities into a number of meaningless components. All pixels with grey level value g, such that $\theta_{\mathrm{i}}<\mathrm{g} \leq \theta_{\mathrm{f}}$, can, in principle, be either foreground or background pixels. In particular, pixels having the same grey level in different parts of the image can be differently classified. The decision on whether they belong to foreground or background is taken during segmentation.

We iterate a process, based on non-topological erosion and topological expansion, which uses different threshold values, automatically obtained increasing the initial value by an increment $\delta$. The increment $\delta$ can be set to any value greater than or equal to one, depending on problem domain. In particular, a small increment is preferable for images where grey level distribution of the foreground is in a large range and also small variations of grey level are significant. 
Let $\theta_{\mathrm{i}}=\theta_{1}, \theta_{2}, \ldots, \theta_{\mathrm{n}}=\theta_{\mathrm{f}}$, be the $\mathrm{n}$ values of the threshold, where the threshold at the $\mathrm{k}$-th iteration is $\theta_{\mathrm{k}}=\theta_{\mathrm{k}-1}+\delta$. In the following, we will describe the process done at the k-th iteration, which consists of two steps, namely i) non-topological erosion and ii) topological expansion. To separate foreground components that result to be merged in a fusion area characterised by a grey value corresponding to the current threshold value, pixels placed in the fusion area among components have to be identified and assigned to the background (i.e., their grey levels have to be lowered to the background value $\theta_{1}$ ). To this purpose, non-topological erosion is accomplished. This is a process that assigns pixels belonging to the border of foreground components to the background, regardless of the topology changes possibly caused by this operation. Border pixels are identified as pixels having at least an edge-neighbour in the background, i.e., pixels with at least one edge-neighbour with grey level equal to $\theta_{1}$. Nontopological erosion is iterated until border pixels are found. In particular, at the k-th iteration, pixels that are possibly placed in a fusion area are those with grey level $\mathrm{g}$ such that $\theta_{1}<\mathrm{g} \leq \theta_{\mathrm{k}}$. Among pixels with grey level in the current range of values, only those that, when inspected, result to be border pixels are assigned to the background.

Border pixels are examined in increasing grey level order and have their grey levels lowered to the background value $\theta_{1}$, regardless on whether connectedness is preserved in their neighbourhood. However, the number of connected components of foreground pixels, i.e., with grey level greater than $\theta_{1}$, in the neighbourhood of any removed pixel is counted. In fact, if more than one component is found, this indicates that the removed pixel was actually a fusion point among foreground components. If this is the case, a fragmentation counter $f_{c}$, initially set to zero, is increased by one. Each removed pixel is orderly stored in a suitable list $\mathrm{L}$, to keep track of its coordinates and grey level. Removal is repeated on the image as far as border pixels with grey level in the current range of values are found. At the end of this process, the counter $f_{c}$ is examined. If $f_{c}=0$, no fusion occurred for the current threshold, and all removed pixels should be recovered to preserve the information contents of the image. In turn, if $f_{c} \neq 0$, some fusion actually occurred. Of course, not all pixels removed at the k-th iteration were actually responsible of fusion among components. Those far from the fusion area should be recovered. Thus, in any case, before proceeding to the successive iteration with a new threshold, topological expansion is performed, to restore size and shape of foreground components, which have been modified by nontopological erosion. Of course, recovery of foreground pixels is done in such a way to still maintain as separated the components that have been singled out. The process is straightforward if no fusion occurred at $\theta_{k}$. All pixels previously stored in L are newly assigned their initial grey level in the image G.

In turn, if some fusion occurred, care should be taken when setting on $G$ the pixels from $L$. To this purpose, pixels of $L$ are examined in the opposite order with respect to their insertion order in L. For the current pixel $\mathrm{p}$, the number of components of foreground pixels (i.e., pixels with grey level greater than $\theta_{1}$ ) is counted after $p$ is restored in G. If only one component is found, $p$ is just assigned on $G$ its initial grey level, as no fusion occurred in $\mathrm{p}$. Otherwise, $\mathrm{p}$ is also suitably marked in $\mathrm{G}$ to point out that it was actually a fusion point.

Once all pixels of the list $\mathrm{L}$ have been processed and restored in $\mathrm{G}$, the fusion marker is iteratively propagated onto successive neighbours of the marked pixels, provided that these neighbours are pixels that have been recovered from L. Marker propagation is performed to identify all pixels in the fusion area. Unfortunately, 
marker propagation may include in the fusion area more pixels than those whose removal is strictly necessary to separate foreground components. Since we aim at changing the status of the lowest possible number of pixels, a suitable process is performed to reduce the thickness of the set of marked pixels, so as to better preserve the original contents of G. Finally, all pixels still marked as fusion points are set to $\theta_{1}$, which results in the separation of foreground components at $\theta_{\mathrm{k}}$.
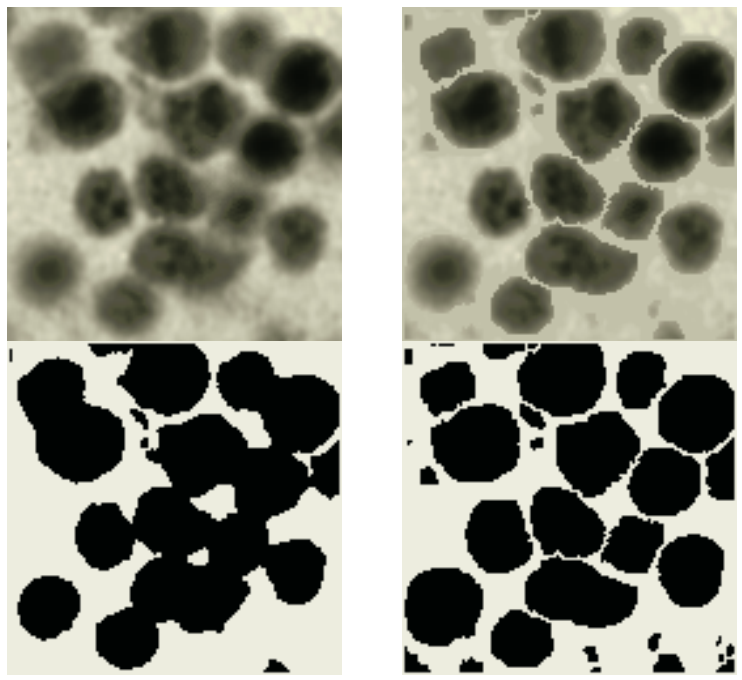

Fig. 1. Top: the input grey level image $G$, left, and the resulting image $G_{1}$, right. Bottom: binary images obtained from $\mathrm{G}$, left, and $\mathrm{G}_{1}$, right.

The threshold $\theta_{\mathrm{k}}$ is incremented by $\delta$ and the $(\mathrm{k}+1)$-th iteration of non-topological erosion and topological expansion are performed, to separate foreground parts at the successive threshold value. The process is iterated until all threshold values are used.

The effect of our segmentation process can be seen in Fig. 1. The two images in Fig.1 top, respectively show the input grey level image $G$ (left) and the image $G_{1}$ (right), resulting after non-topological erosion and topological expansion. The two images in Fig. 1 bottom, show the binarizations of $G$ and $G_{1}$. In the image to the right pixels with grey level equal to or smaller than $\theta_{1}$ are set to 0 and the remaining pixels are set to 1 , to point out which are the foreground components singled out by our process; in the image to the left a threshold value greater than $\theta_{1}$ has been used for the binarization so as to favour segmentation, which however is not satisfactory. The values for $\theta_{1}, \theta_{\mathrm{n}}$ and $\delta$ have been heuristically selected, based on the image domain. In particular, $\theta_{1}=70, \theta_{\mathrm{n}}=170$ and $\delta=5$.

\section{A Topology Preserving Multi-scale Representation}

As pointed out in Introduction, our segmentation method can find a useful application in multi-scale image analysis. Having a multi-scale image representation is of interest in a number of tasks, e.g., [7-11], since successively condensed representations of the information in the input image are available and the user can often work with a re- 
duced data set, at low resolution, which still provides a reasonable representation of the most relevant regions.

Image pyramids are among the most common multi-scale representation systems [12]. Both continuous and discrete methods to build pyramids can be found in the literature. We here describe a discrete method to build a topology preserving pyramid.

Two important features to use pyramids in the framework of image analysis are shift invariance and topology preservation [13]. Representations at low resolution can differ significantly from the input image, when this is shifted or, better, when the partition grid used to performed decimation, is shifted along the image. It is, in fact, quite obvious that a different position of the partition grid generally leads to a significantly different image pyramid. Moreover, the mechanism used to build the pyramid can significantly alter, besides geometry and shape, also the topology of the input image, so that the representative power of lower resolution images would become questionable. Topology preservation is not considered in methods based on filters, $[14,15]$, while is taken into account in generation methods of irregular pyramids. In this latter case, however, the father-child relation is lost or altered, [16, 17]. Our aim is to obtain an almost shift invariant and topology preserving grey level pyramid, without altering or destroying the father-child relation, and based on a regular tessellation. We introduced in [18] a method to generate a pyramid, starting from a binary image, which is shift invariant and topology preserving, within the limits of decreasing resolution. The method here illustrated is an extension of the one in [18] to the case of grey level images.

To build the pyramid, a recursive subdivision into quadrants of the $2^{\mathrm{n}} \times 2^{\mathrm{n}}$ grey level image, $G_{1}$, is performed. At each recursion step, resolution decreases by four and the process terminates when the image including one single pixel is built. This is the general scheme, but is obvious that resolution levels with less than 16 pixels in each dimension can be disregarded, as they would not be useful in shape analysis tasks. Thus, the pyramid construction will actually end as soon as the $2^{4} \times 2^{4}$ image is built. The base of the pyramid is the input image $G_{1}$ at full resolution $(128 \times 128$, for the example shown in this paper), the next level of the pyramid represents the image $\mathrm{G}_{2}$ at a uniformly lower resolution $(64 \times 64)$ and so on. The apex of the pyramid is the $16 \times 16$ image $\mathrm{G}_{4}$.

Pyramid construction is accomplished by using the image $G_{1}$ segmented by the process described in Section 2. Foreground components are assigned an identity label by means of connected component labelling. To build $\mathrm{G}_{2}$, a partition grid is superimposed onto $\mathrm{G}_{1}$. The grid consists of non-overlapping blocks of $2 \times 2$ pixels. Each block originates one pixel in $\mathrm{G}_{2}$. The four pixels in a block are the children, and the pixel corresponding to the whole block in $\mathrm{G}_{2}$ is the parent. The grey level value of a parent pixel is computed by taking into account number, positions and grey levels of its four children. The label identifying the foreground component to which children belong is also transferred to the parent pixel. When children belong to different components, a majority rule is used to decide the label for the parent pixel.

Depending on the position of the partition grid over $G_{1}$, a pixel $p$ in the image $G_{1}$ belongs to one out of four possible blocks, including three out of the eight neighbours of $\mathrm{p}$. In turn, each neighbour of $\mathrm{p}$ belongs to a different number of blocks, depending 
on its position with respect to $\mathrm{p}$. Each edgeneighbour of $\mathrm{p}$ belongs to two different $2 \times 2$ blocks including $\mathrm{p}$, while each vertexneighbour belongs to exactly one such $2 \times 2$ block. See Fig. 2.

The decimation filter is a $3 \times 3$ mask of weights, with central value 4 , edgeneighbours valued 2 and vertex-neighbours valued 1 . This multiplicative mask is centred on each pixel $p$ of $\mathrm{G}_{1}$ with even coordinates
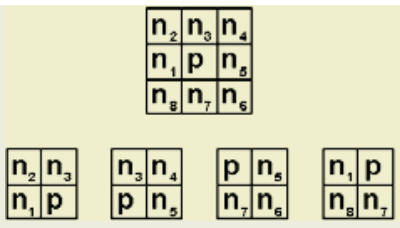

Fig. 2. The eight neighbours of $\mathrm{p}$, top, and the four $2 \times 2$ blocks that can be obtained by shifting the partition grid.

$(2 \mathrm{j}, 2 \mathrm{k})$, and the value of the corresponding parent pixel $\mathrm{p}^{\prime}$ with coordinates $(\mathrm{j}, \mathrm{k})$ in $\mathrm{G}_{2}$, is computed. The image $\mathrm{G}_{2}$ is then re-scaled by linearly re-scaling its pixels to the range $\{0,255\}$ and the mask is applied to build $\mathrm{G}_{3}$, and so on.

Since the multiplicative mask takes into account all possible $2 \times 2$ non-overlapping blocks, the resulting pyramid will be almost shift invariant. As concerns topology preservation, a decision is necessary on whether foreground or background components have to be favoured, since when resolution decreases it can be impossible to preserve both. We favour foreground components.

We use a landscape representation of the grey level image, where the grey level of a pixel is interpreted as the height in a topographic map. In this way, the image $\mathrm{G}_{1}$ can be interpreted as consisting of a number of islands, some of which may include lakes and craters. Islands of $\mathrm{G}_{1}$ close to each other can merge into a unique component in $\mathrm{G}_{2}$, lakes and craters can be transformed into less deep craters, and new lakes can appear, due to deformation of bays. To preserve topology in the limits of reduced resolution, we use $\mathrm{G}_{1}$ as a reference image and open suitable canals at lower resolutions to separate islands erroneously merged into a unique component. We also open spurious craters rather than filling them. In this way, the effect produced by the modified value distribution better resembles that of the original image. This opening is done at each resolution level before computing the next pyramid level, so as to avoid chain reaction effects.

To open canals in $\mathrm{G}_{2}$, we check for each foreground pixel if a neighbour exists, having different identity label. In the affirmative, the pixel with the lowest grey level (and, hence, the lowest relevance) in the adjoining pair is assigned to the background.

A crater is a set of pixels with value strictly smaller than all values of the pixels on the 8-connected rim of the crater. Craters and lakes which do not correspond to craters and lakes in the full resolution image are interpreted as spurious entities, and are transformed into bays by iteratively identifying their bottom and by assigning to the rim of the bottom the grey level of the bottom.

The pyramid built for the grey level image shown in Fig.1, is illustrated in Fig. 3. Only levels $64 \times 64,32 \times 32$, and $16 \times 16$ are

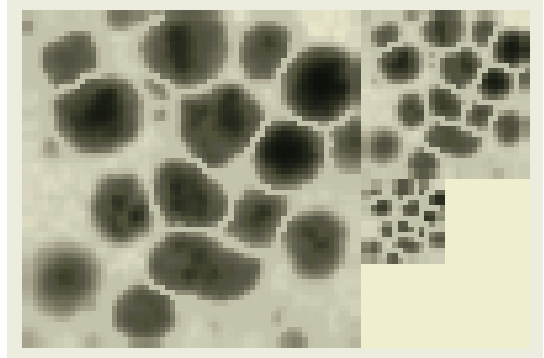

Fig. 3. The pyramid built for the grey level image shown in Fig.1. A zoom factor 2 is used. 
shown, since level $128 \times 128$ is the image in Fig. 1 top right. A zoom factor 2 has been used to make visible these resolution levels. We note that foreground components are preserved also at low resolution.

\section{Conclusion}

We have introduced a method to single out foreground components, which is suitable in the framework of multi-scale grey level image analysis.

Foreground components detection is done by using an iterated process, based on non-topological erosion followed by topological expansion. A multi-scale representation is then built starting from this image. To limit translation dependency, the four different images that would be generated by shifting, in the four possible positions, the partition grid used to build lower resolutions are combined. Topology preservation is favoured by identifying on the highest resolution image all foreground components. This allows us to open canals to separate, at lower resolution, foreground parts that constituted individual entities at the highest resolution level. Naturally, topology is preserved compatibly with the resolution, through lower resolution pyramid levels.

The method we have developed was mainly intended to treat biological specimens, as the one shown in Fig.1. However, we think that our method could also be used in other fields, e.g., for document analysis. More in general, the method is adequate for application areas dealing with grey level images in which foreground components can be distinguished from the background.

\section{References}

1. Brink A.D., Thresholding of digital images using two-dimensional entropies, Pattern Recognition 25-8 (1992) 803-808.

2. O'Gorman L., Binarization and multithresholding of document images using connectivity, Graphical Models and Image Processing (1994) 56-6.

3. Papamarkos N., Strouthopoulos C., Andreadis I., Multithresholding of color and gray-level images through a neural network technique, Image and Vision Computing 18-3 (2000) 213222.

4. Vincent L., Soille P., Watersheds in digital space: an efficient algorithm based on immersion simulations. IEEE Trans. PAMI 13- 6 (1991) 583-598.

5. Beucher S., Meyer F., The morphological approach to segmentation: the watershed transformation, in Mathematical Morphology in Image Processing, E. Dougherty ed., Marcel Dekker, Chap. 12 433-481 (1993).

6. Bleau A., Leon L.J., Watershed-based Segmentation and Region Merging, Computer Vision and Image Understanding 77 (2000) 317-370.

7. Yacoub S.B., Jolion J.M:, "Hierarchical line extraction", Proceedings of IEEE Conf. on Vision, Image and Signal Processing 142 (1995) 7-14.

8. Tan C.L., Loh S.K.K., "Efficient edge detection using hierarchical structures", Pattern Recognition, 26-1 (1993) 127-135.

9. Brzakovic D., Luo X.M., Brzakovic P., An approach to automated detection of tumors in mammograms. IEEE Transactions on Medical Imaging 9-3 (1990) 233-241.

10. Tan C.L., Ng P.O., Text extraction using pyramid. Pattern Recognition 31-1 (1998) 63-72.

11. Bosworth J., Koshimizu T., Acton S.T., Automated segmentation of surface soil moisture from landsat TM data, Proceedings of IEEE Southwest Symposium on Image Analysis and Interpretation (1998) 70-74.

12. Rosenfeld, A. (Ed.), Multiresolution Image Processing and Analysis. Springer-Verlag, Berlin (1984). 
13. Bister M., Cornelis J., Rosenfeld A., A critical view of pyramid segmentation algorithms, Pattern Recognition Letters 11- 9 (1990) 605-617.

14. Burt P.J., The Pyramid as a Structure for Efficient Computation, in A. Rosenfeld, (ed.) Multiresolution Image Processing and Analysis, Springer-Verlag, Berlin (1984).

15. Greenspan H., Belongie S., Goodman R., Perona P., Rakshit S., Anderson C.H., Overcomplete steerable pyramid filters and rotation invariance, Proceeding IEEE Computer Vision and Pattern Recognition, Seattle, Washington (1994) 222-228.

16. Arman F., Pearce J.A., Unsupervised classification of cell images using pyramid node linking, IEEE Trans Biomedical Engineering 37- 6 (1990) 647-50.

17. Montanvert A., Meer P., Rosenfeld A., Hierarchical Image Analysis Using Irregular Tessellations, IEEE Trans. PAMI 13 - 4 (1991) 307-316.

18. Borgefors G., Ramella G., Sanniti di Baja G., Shape and topology preserving multi-valued image pyramids for multi-resolution skeletonization, Pattern Recognition Letters 22 (2001) 741-751. 